\title{
Effect of neoadjuvant chemotherapy regimen on relapse-free survival among patients with breast cancer achieving a pathologic complete response: an early step in the de-escalation of neoadjuvant chemotherapy
}

\author{
Anna Weiss ${ }^{1}$, Sami I. Bashour ${ }^{2}$, Kenneth Hess ${ }^{3}$, Alastair M. Thompson ${ }^{4}$ and Nuhad K. Ibrahim²*
}

\begin{abstract}
Background: Patients with breast cancer who have a pathologic complete response $(\mathrm{pCR})$ to neoadjuvant chemotherapy (NACT) have improved survival. We hypothesize that once pCR has been achieved, there is no difference in subsequent postsurgical recurrence-free survival (RFS), whichever NACT regimen is used.

Methods: Data from patients with breast cancer who achieved PCR after NACT between 1996 and 2011 were reviewed. RFS was estimated by the Kaplan-Meier method, and differences between groups were assessed using log-rank testing. Cox proportional hazards regression analysis adjusted for age, menopausal status, stage, grade, tumor subtype, and adjuvant endocrine HER2-targeted radiation treatment.

Results: Among 721 patients who achieved pCR after NACT, 157 (21.8\%) were hormone receptor-positive (HR), 310 (43.3\%) were HER2-amplified, and 236 (32.7\%) were triple-negative; 292 (40.5\%) were stage IIA, 153 (21.2\%) were stage IIB, 78 (10.8\%) were stage IIIA, 66 (9.2\%) were stage IIIB, and 132 (18.3\%) were stage IIIC. Most patients (367 [50.9\%]) had been treated with adriamycin-based chemotherapy plus taxane $(A+T), 56$ (7.8\%) without taxane (A no T), 227 (31.5\%) with HER2-targeted therapy, and 71 (9.8\%) provider choice. Median follow-up was 7.1 years. Adjuvant chemotherapy was employed in 196 (27\%) patients, adjuvant endocrine in 261 (36\%), and adjuvant radiation in the majority (559 [77. 5\%]). There was no statistically significant difference in RFS by NACT group. Adjusted RFS hazard ratios, comparing each treatment with the reference group A + T, were 1.25 (95\% Cl 0.47-3.35) for A no T, 0.90 (95\% Cl 0.37-2.20) for HER2-targeted therapy, and 1.28 (95\% Cl 0.55-2.98) for provider choice.
\end{abstract}

Conclusions: These data suggest that postsurgical RFS is not significantly influenced by the choice of NACT or cancer subtype among patients achieving pCR.

Keywords: Neoadjuvant chemotherapy, Pathologic complete response, De-escalation of chemotherapy, Breast cancer, Survival after pCR

\footnotetext{
* Correspondence: nibrahim@mdanderson.org

This abstract was presented at the American Society of Clinical Oncology annual meeting June 4, 2017, as a poster and was awarded a Conquer Cancer Merit Award. The abstract from this presentation was published elsewhere previously.

${ }^{2}$ Department of Breast Medical Oncology, University of Texas MD Anderson

Cancer Center, 1155 Pressler Street CPB5.3540, Houston, TX 77030, USA

Full list of author information is available at the end of the article
} 


\section{Background}

Neoadjuvant chemotherapy (NACT), originally used to downstage breast cancer, may achieve a pathologic complete response $(\mathrm{pCR})$ in a proportion of patients, with no residual invasive disease in the breast or nodes [1]. The residual cancer burden (RCB) present after NACT reflects response to chemotherapy and predicts survival. Consequently, low (RCB I) or no residual disease ( $R C B$, defined as $\mathrm{pCR}$ ) is associated with improved survival compared with more residual disease (RCB II or III), a relationship that holds true across breast cancer subtypes [1-4]. Large randomized clinical trials have shown that more recent NACT regimens have provided increased PCR rates $[5,6]$, and some trials have shown resultant improved survival $[1,7]$. Although the timing of chemotherapy and use of NACT have not historically impacted overall survival (OS) [8-13], important therapeutic information may be gained by evaluating tumor response to chemotherapy [14, 15]. Although an imperfect tool [1], pCR has been approved by the U.S. Food and Drug Administration as an acceptable surrogate endpoint for key clinical trials [16-19].

The relationship between complete pathologic response to NACT and survival in triple-negative breast cancer has been used for statistical modeling and clinical trial power calculations [20]. This modeling assumes that all patients with a pCR have similar survival, but this assumption of NACT regimen equivalence has not been examined. The objectives of this study were to determine if a specific treatment regimen may be associated with preferential survival in patients who achieve a pCR and to identify risk factors for recurrence among patients with a pCR.

\section{Methods}

Patients diagnosed with primary breast cancer on the basis of cytology or histopathology needle biopsy, treated between 1996 and 2011 with various regimens of NACT at a single high-volume cancer center, were reviewed using a prospectively maintained electronic database. $\mathrm{Pa}-$ tients were considered eligible for review if, after NACT, they achieved pCR on the basis of final surgical pathology. pCR in this study was defined as eradication of invasive disease in both the breast and lymph nodes (T0/Tis, N0), the definition used at our institution, consistent with the large pooled analysis performed by Cortazar et al. [1]. Patients were excluded if any part of their chemotherapy regimen was received at an outside institution or after definitive breast surgery. This singleinstitution study was approved by the University of Texas MD Anderson Cancer Center Institutional Review Board.

Patients were divided into the following four major chemotherapy regimens: adriamycin-based chemotherapy alone (A no T), adriamycin-based chemotherapy plus taxane $(\mathrm{A}+\mathrm{T})$, HER2-targeted therapy $(\mathrm{AT}+\mathrm{HER} 2)$, or provider choice $(\mathrm{PC})$. Provider choice regimens were all others; that is, all included a taxane base, but with the addition of clinical trial agents such as capecitabine or bevacizumab. Duration of NACT was calculated as the date of first chemotherapy treatment to the date of surgery, including a typical 4- to 6-week window between completion of NACT and surgery. There were three NACT duration cohorts: $\leq 4$ months, $>4$ and $\leq 7$ months, and $>7$ months. The group treated for $\leq 4$ months was treated early in the study time frame according to a departmental algorithm that included four cycles only (most commonly doxorubicin, cyclophosphamide, and 5-fluorouracil; or fluorouracil, adriamycin, and cyclophosphamide $[\mathrm{FAC}]$ ). Recurrence-free survival (RFS) of pCR patients was compared between these four regimens, agnostic to the details of drugs received. Recurrence and death data were collected by in-depth chart review. The RFS was estimated by the KaplanMeier method; calculations were made at 5 years; and the differences between groups were assessed using log-rank testing. Values were statistically significant if $P<0.05$. RFS was next compared by breast cancer tumor subtype (hormone receptor [HR]-positive, HER2-amplified, and triple-negative [TN]), and then within each of these subtypes the RFS was again calculated and compared for the four specific NACT regimens. RFS was next calculated by age group (21$40,41-60$, and $>60$ years old), stage (IIA, IIB, IIIA, IIIB, and IIIC), and grade (1-3). Age groups were determined by quartiles, with patients aged 21-40 years constituting the youngest quartile and those aged $>60$ years the oldest quartile [21]. Last, the RFS was compared by length of neoadjuvant chemotherapy.

Adjuvant treatments were analyzed via landmark analysis [22] with time point zero set to 6 months following surgery to allow for completion of adjuvant treatments (adjuvant chemotherapy, radiation). The landmark cutoff did not include completion of endocrine or anti-HER2 therapy. Patients who were censored or failed (recurrence or death within 6 months of surgery) were omitted. This approach allowed adjuvant treatments to be included as baseline factors in the analysis. Patients omitted were those who failed as described $(n=4)$ or if clinicopathologic details (age, grade, stage, HR or HER2 status, adjuvant treatments received) were missing $(n=75)$. Too few patients had neoadjuvant radiation treatment $(n=1)$ or neoadjuvant endocrine treatment $(n=6)$ for landmark analysis. Cox proportional hazards regression analysis adjusted for NACT (using treatment arm A + $\mathrm{T}$ as the reference group), age (reference group 41- to 60-year-olds), menopausal status (reference group 
premenopausal), stage (reference group stage IIA), grade (reference group grade 1), tumor subtype (reference group TN breast cancer), and adjuvant treatments (reference group for each therapy was no treatment received).

\section{Results}

\section{Patient characteristics}

A total of 921 patients were identified over the 15year period, 200 of whom received part of their chemotherapy treatment at an outside institution and were excluded, leaving 721 patients in the survival analyses (Fig. 1). Of these 721 patients, 664 (92\%) had ductal histology (Table 1). The youngest quartile of age, 21-40 years, included 154 patients (21.4\%), the group ages 41-60 years included 449 patients (62.3), and the group aged $>60$ years included 118 patients (16.4\%). Race breakdown included 423 (59\%) white patients, 136 (19\%) Hispanic patients, 109 (15\%) black patients, 45 (6\%) Asian patients, and 8 (1\%) categorized as unknown/other. A majority of patients (389 [54\%]) were postmenopausal. Most patients $292(40.5 \%)$ were stage IIA, $153(21.2 \%)$ were stage IIB, 78 (10.8\%) were stage IIIA, 66 (9.2\%) were stage IIIB, and $132(18.3 \%)$ were stage IIIC. By tumor subtype, 157 (21.8\%) were HR-positive, 310 (43.3\%) were HER2-amplified, $236(32.7 \%)$ were $\mathrm{TN}$, and 18 (2.5\%) were of unknown type. Of the 310 HER2amplified patients, 128 were $\mathrm{HR}+, 178$ were $\mathrm{HR}-$, and 4 had unknown HER2 status. Patients were grouped into four chemotherapy regimens: 367 (50.9\%) patients treated with $\mathrm{A}+\mathrm{T}, 56(7.8 \%)$ treated with $\mathrm{A}$ no T, $227(31.5 \%)$ treated with AT + HER2, and 71 (9.8\%) treated with PC (Fig. 1). HER2+ patients were treated both before and after the standard use of HER2-targeted therapies, 247 received HER2-targeted therapy (20 of these in the PC group), and 60 did not. Survival was also examined within this subset. The median length of NACT was 6.0 months, ranging from $<1$ month to 12 months. The majority of patients with shorter duration of NACT $(<4$ months) received four cycles of FAC or fluorouracil, epirubicin, and cyclophosphamide (43 of 64 [67\%]). Other regimens included adriamycin plus a taxane (10 of 64 [16\%]); taxane alone (6 of 64; [9\%]); FAC + taxane (3 of 64; [5\%]); adriamycin and cyclophosphamide (1 of 64; [2\%]); and taxotere, carboplatin, and herceptin (1 of 64 [2\%]). In this group, 61 of 64 patients $(95 \%)$ completed their planned regimen

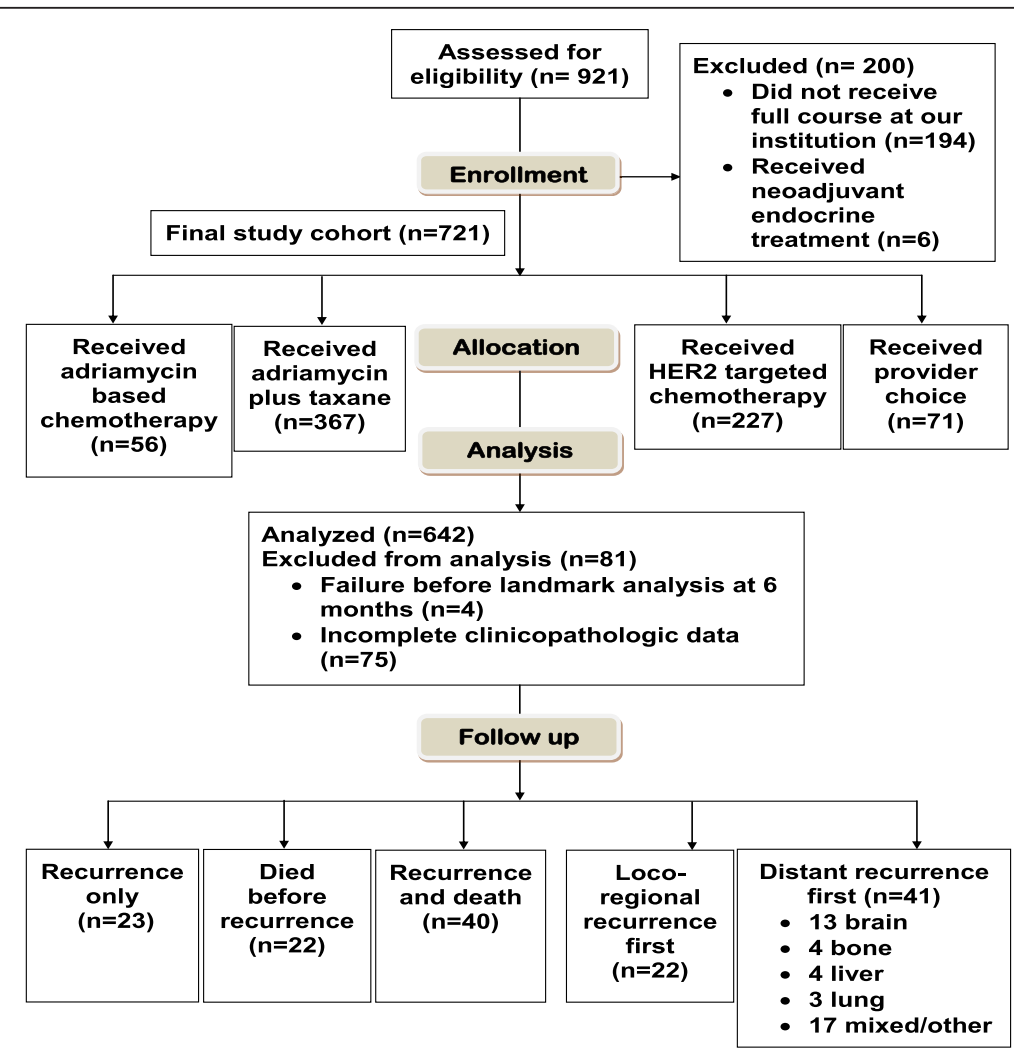

Fig. 1 Consolidated Standards of Reporting Trials (CONSORT) diagram depicting the entire study cohort. There were 921 patients with a pathologic complete response at our institution during the study period. Two hundred of these patients received part of their chemotherapy at an outside institution. A total of 721 patients were included in survival analyses, and 642 in were included the multivariate analysis 
Table 1 Patient characteristics of the entire cohort

\begin{tabular}{|c|c|c|}
\hline Characteristics & No. of patients $(N=721)$ & $\%$ \\
\hline \multicolumn{3}{|l|}{ Breast cancer type } \\
\hline Invasive ductal & 664 & 92 \\
\hline Invasive lobular & 9 & 1 \\
\hline Mixed ductal/lobular & 14 & 2 \\
\hline Breast cancer, NOS ${ }^{a}$ & 16 & 2 \\
\hline Other & 18 & 3 \\
\hline
\end{tabular}

Age, years

$\begin{array}{ll}21-40 & 154 \\ 41-60 & 449 \\ >60 & 118\end{array}$

Race

$\begin{array}{ll}\text { White } & 423 \\ \text { Hispanic } & 136 \\ \text { Black } & 109 \\ \text { Asian } & 45 \\ \text { Other } & 8\end{array}$

Menopausal status

Unknown

Premenopausal

Pregnant

3

Perimenopausal

Postmenopausal

Clinical stage

$\begin{array}{ll}\text { IA } & 292 \\ \text { IIB } & 153 \\ \text { IIA } & 78 \\ \text { III } & 66 \\ \text { IIC } & 132\end{array}$

Estrogen receptor status

$\begin{array}{ll}\text { Unknown } & 15 \\ \text { Negative } & 454 \\ \text { Positive } & 252\end{array}$

Progesterone receptor status

$\begin{array}{ll}\text { Unknown } & 19 \\ \text { Negative } & 526 \\ \text { Positive } & 176\end{array}$

HER2 status

$\begin{array}{ll}\text { Negative } & 286 \\ \text { Positive } & 312 \\ & 123\end{array}$

Equivocal

Tumor subtype

$\begin{array}{ll}\text { Unknown } & 18 \\ \text { HR } & 157 \\ \text { TN } & 236\end{array}$

40
Table 1 Patient characteristics of the entire cohort (Continued)

\begin{tabular}{lll}
\hline Characteristics & No. of patients $(N=721)$ & $\%$ \\
\hline HER2-amplified & 310 & 43 \\
HR+ & 128 & 41 \\
HR- & 178 & 57 \\
Unknown HR & 4 & 1 \\
Neoadjuvant chemotherapy & & \\
A no T & 56 & 8 \\
A + T & 367 & 51 \\
AT + HER2 & 227 & 31 \\
PC & 71 & 10
\end{tabular}

HER2+ patient regimens

HER2-containing regimen $\quad 247 \quad 80$

No HER2

60

20

Length of NACT therapy

0-4 months

64

570

79

$4 \& 7$ months

87

12

Neoadjuvant hormonal therapy

Yes

12

0

No

715

100

Neoadjuvant radiation therapy

Yes

0

No

100

Adjuvant chemotherapy

Yes

27

No

73

Adjuvant endocrine therapy

Yes

36

No

261

64

Adjuvant radiation therapy

Yes

559

78

No

22

Outcomes

Progression

Death (any cause)

Progression + death

162

Death without progression

63

62

9

9

6

Site of metastasis

Distant first

Brain-only

Bone-only

Liver-only

Lung-only

Mixed/other

8

10

57

51

80
9
9
12

00


Table 1 Patient characteristics of the entire cohort (Continued)

\begin{tabular}{cll}
\hline Characteristics & No. of patients $(N=721)$ & $\%$ \\
\hline Local first & 11 & 17 \\
Chest wall-only & 1 & 9 \\
Ipsilateral breast-only & 10 & 91 \\
Both local and distant & 11 & 17 \\
Concurrent $^{\mathrm{a}}$ & 6 & 55 \\
Sequential & 5 & 45
\end{tabular}

Abbreviations: NOS Not otherwise specified, HR Hormone receptor; TN Triple-negative breast cancer, $A$ no $T$ Adriamycin-based therapy alone, $A+$ $T$ Adriamycin plus taxane, $A T+H E R 2$ HER2-targeted therapy, $P C$ Provider choice, NACT Neoadjuvant chemotherapy

${ }^{a}$ Concurrent local and distant metastases were diagnosed within 1 month of each other

without issue. Two patients stopped NACT after three cycles because of neutropenia, and one patient proceeded to surgery after only two cycles per the patient's request.

Definitive surgical treatments included total mastectomy and axillary lymph node dissection (ALND) in 256 (35.5\%), total mastectomy and sentinel lymph node biopsy (SLNB) in 46 (6.4\%), skin-sparing mastectomy and ALND in 44 (6.1\%), skin-sparing mastectomy and SLNB in 51 (7.1\%), partial mastectomy and ALND in 176 (24.4\%), partial mastectomy and SLNB in 130 (18\%), 1 radical mastectomy (0.1\%), 2 wide local excisions after breast conservation (0.3\%), 5 excisional biopsies (0.7\%), and 10 ALND only (1.4\%). Most patients (708 [98.2\%]) had free surgical margins at initial operation. Adjuvant chemotherapy was employed in 196 (27\%) patients, endocrine therapy in 261 (36\%), and adjuvant radiation in the majority (559 [77.5\%]).

\section{Survival analysis}

Median follow-up for those without recurrence was 7.1 years $(0.02-16.7)$, and median follow-up by the reverse
Kaplan-Meier method for those with recurrence was 7.4 years (5th-95th percentiles 3.2-12.3). There were a total of 85 events of recurrence or death: 40 patients had recurrence and died, 23 had recurrence only, and 22 died without recurrence. Forty-one patients (65\%) with recurrence had recurrence at a distant site first (13 [31.7\%] brain only, 4 [10\%] bone only, 4 [10\%] liver only, 3 [7\%] lung only, and 17 [41\%] mixed/other). Nine of the 13 brain-only metastases occurred in HER2 amplified patients.

There was no statistically significant difference in RFS by treatment group (Fig. 2) (log-rank test $P=0.45)$. Patients treated with A no T $(n=56)$ had a 5 -year RFS of 93\% (95\% CI 86-100\%), $\mathrm{A}+\mathrm{T}(n=367)$ 91\% (88-94\%), $\mathrm{AT}+\mathrm{HER} 2(n=227) 93 \%(89-96 \%)$, and PC $(n=71)$ 85\% (78-94\%) (Table 2, Fig. 2). Patients who were HER2 + who achieved a pCR and received HER2-targeted therapy had 5-year RFS similar to those who did not (94\% received versus $90 \%$ no HER2 therapy; $P=0.19)$.

By tumor subtype, HR-positive patients $(n=143)$ had a 5 -year RFS of 91\%, HER2-amplified patients $(n=312)$ $92 \%$, and TN $(n=216) 90 \%(P=0.26)$. Patients who had HER2 + disease had similar survival by HR status $(94 \%$ $\mathrm{HR}+$ versus $92 \% \mathrm{HR}-; \quad P=0.12$ ). Within each tumor subtype, RFS by NACT regimen was compared. There was no significant difference between significant NACT regimen within HER2-amplified disease $(P=0.42)$ or TN disease $(P=0.59)$ (Table 2 , Fig. 3$)$. The NACT regimen sample sizes within HR-positive disease were too small to accurately calculate the log-rank test.

By unadjusted analyses, the RFS of 21- to 40-yearolds $(n=154)$ was $89 \%, 41$ - to 60 -year-olds $(n=449)$ was $93 \%$, and 61 - to 90 -year-olds $(n=118)$ was $88 \%$ (Fig. 4). RFS by stage was $93 \%$ for stage IIA patients $(n=292), 91 \%$ for stage IIB $(n=153), 95 \%$ for stage

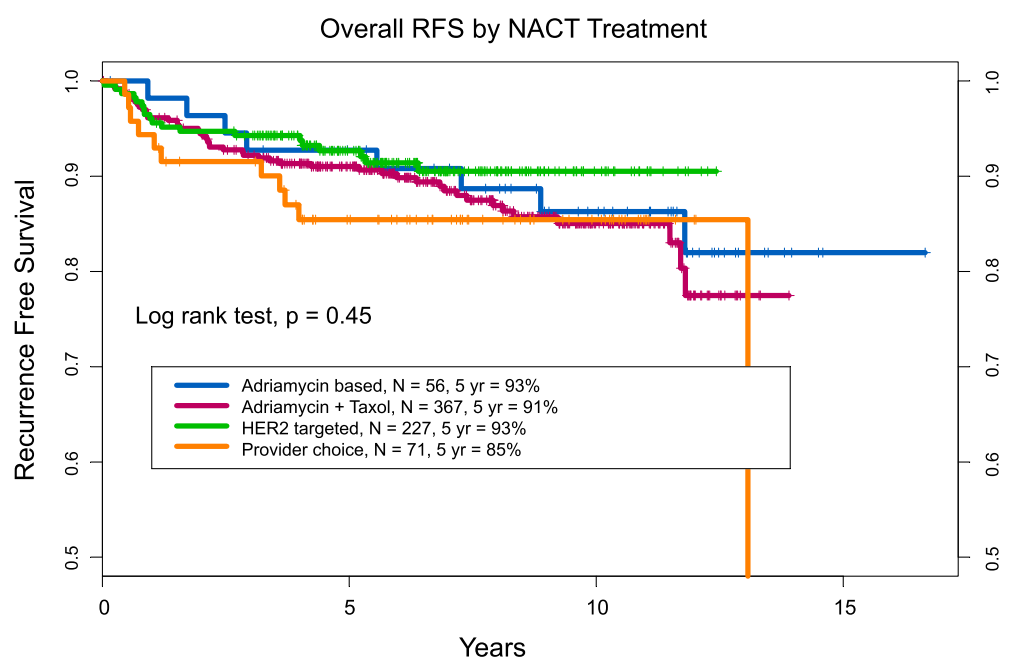

Fig. 2 Recurrence-free survival for the entire cohort, compared between the four neoadjuvant chemotherapy (NACT) treatment regimens 
Table 2 Recurrence-free survival for the total cohort and by tumor subtype

\begin{tabular}{|c|c|c|c|c|c|c|c|c|}
\hline \multirow{2}{*}{$\begin{array}{l}\text { Treatment } \\
\text { group }\end{array}$} & \multicolumn{2}{|c|}{ Overall $(\mathrm{N}=721, P=0.45)$} & \multicolumn{2}{|c|}{$\mathrm{HR}(n=143, P=\mathrm{NA})$} & \multicolumn{2}{|c|}{ HER2-amplified $(n=312, P=0.42)$} & \multicolumn{2}{|c|}{$\operatorname{TN}(n=216, P=0.59)$} \\
\hline & No. of patients & 5 -yr RFS (\%) & No. of patients & 5-yr RFS (\%) & No. of patients & 5-yr RFS (\%) & No. of patients & 5-yr RFS (\%) \\
\hline A no $T$ & 56 & $93(86-100)$ & & $-^{\mathrm{a}}$ & & $--^{\mathrm{a}}$ & 22 & $86(73-100)$ \\
\hline$A+T$ & 367 & $91(88-94)$ & 124 & $92(87-97)$ & 55 & 89 (80-98) & 170 & $92(87-96)$ \\
\hline $\mathrm{AT}+\mathrm{HER} 2$ & 227 & $93(89-96)$ & & $-{ }^{a}$ & 220 & $93(90-97)$ & & $-{ }^{a}$ \\
\hline PC & 71 & $85(78-94)$ & 11 & $80(58-100)$ & 33 & $91(81-100)$ & 21 & $85(72-100)$ \\
\hline
\end{tabular}

Abbreviations: HR Hormone receptor, $T N$ Triple-negative breast cancer, $A$ no $T$ Adriamycin-based therapy alone, $A+T$ Adriamycin plus taxane, $A T+H E R 2$ HER2-targeted therapy, PC provider choice

${ }^{\mathrm{a}}$ Missing cells $<10$ patients

IIIA $(n=78), 86 \%$ for stage IIIB $(n=66)$, and $87 \%$ for stage IIIC $(n=132)$. RFS for grades 1 and 2 was $86 \%(n=101)$, and for grade $3(n=599)$ it was $92 \%$. Patients who were treated with $\leq 4$ months of NACT had a 94\% 5-year RFS, those treated with $>4$ and $\leq 7$ months $92 \%$, and those treated with $>7$ months $86 \%$ RFS (Additional file 1: Figure S1).

\section{Multivariate analysis}

According to the 6-month landmark analyses, there were no differences attributable to adjuvant chemotherapy (92\% with or without), endocrine therapy (91\% without, 94\% with endocrine therapy), or radiation treatment (92\% with or without). Amongst 21- to 40-year-olds, RFS with A no T was 95\% $(n=19), \mathrm{A}+\mathrm{T} 87 \%(n=81)$,
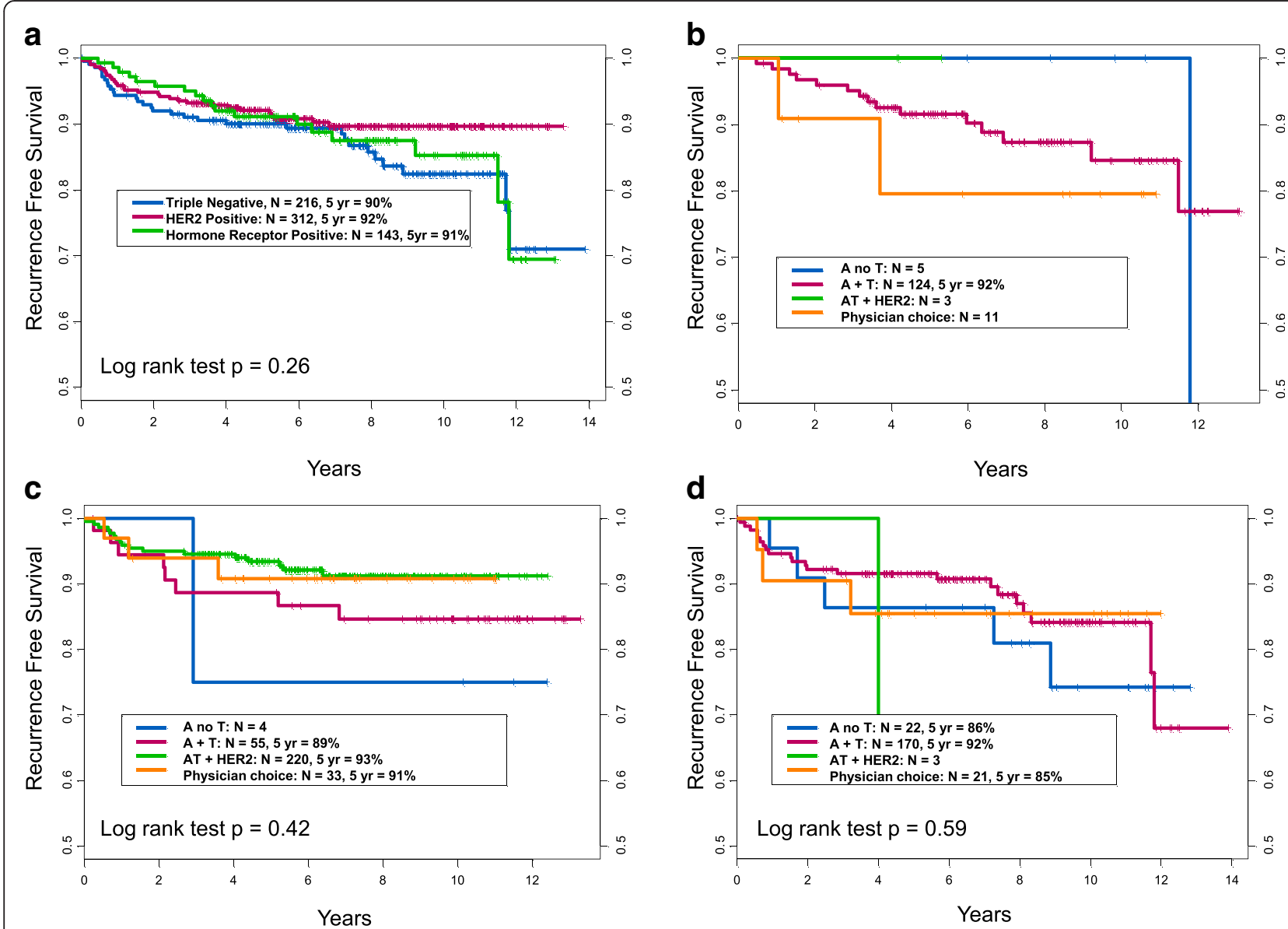

Fig. 3 a Recurrence-free survival (RFS) by tumor subtype. b RFS by neoadjuvant chemotherapy (NACT) treatment regimen within hormone receptor (HR)-positive, HER2-nonamplified patients. c RFS by NACT treatment regimen within HER2-positive patients. d RFS by NACT treatment regimen within triple-negative patients with breast cancer. Some groups were too small to analyze, and survival could not be calculated (b, c, d). Additionally, the log-rank test could not be performed for HR-positive disease (b), owing to small sample size 


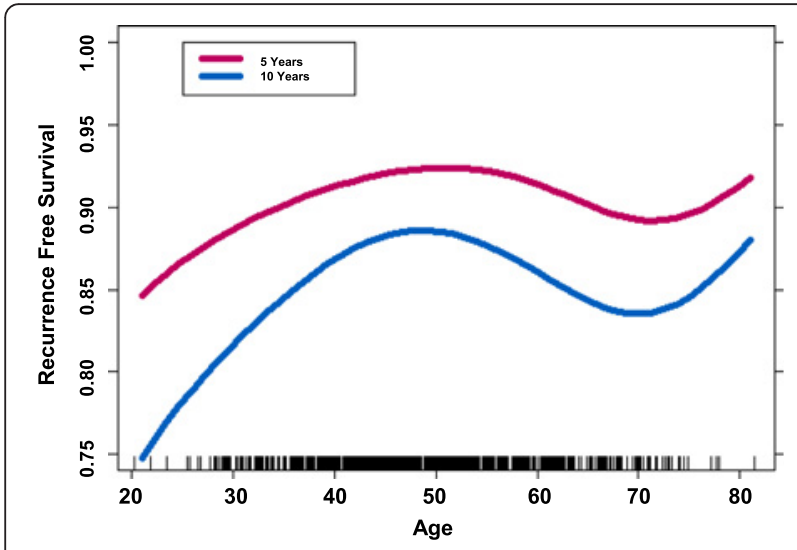

Fig. 4 Recurrence-free survival by age as a continuous variable. There is low survival among very young patients

AT + HER2 88\% $(n=43)$, and PC $(n=11)$ (too small to calculate). Amongst 41- to 60-year-olds, RFS was $90 \%$ for A no T, 93\% A + T, 94\% AT + HER2, and 88\% PC ( $n$ $=32,225,149$, and 43 , respectively). In patients aged $>$ 60 years, RFS was $88 \%$ in patients treated with $\mathrm{A}+\mathrm{T}$ ( $n$ $=61)$ and $94 \%$ in patients treated with treated with AT + HER2 $(n=35)$. There were too few patients in the A no $\mathrm{T}$ and $\mathrm{PC}$ regimens to calculate survival $(n=5$ and 17 , respectively).

Adjusted RFS hazard ratios comparing each treatment with $\mathrm{A}+\mathrm{T}$ were 1.25 (95\% CI $0.47-3.35)$ for $\mathrm{A}$ no $\mathrm{T}$,
0.90 (95\% CI 0.37-2.20) for AT + HER2, and 1.28 (95\% CI 0.55-2.98) for PC (Table 3). For age, the hazard ratio of death, compared with the reference group of 41- to 60year-olds, was 2.00 (95\% CI 1.05-3.82, $P=0.036$ ) for 21 - to 40-year-olds, and 2.04 for 61- to 90-year-olds (95\% CI $1.01-4.15, P=0.048)$. There was a significantly increased risk of death for stage IIIC patients $(2.26,95 \%$ CI 1.11-4.61, $P=0.024)$. There was no significant difference between the hazard ratio of death by menopausal status $(P=0.57)$; grade $(P=0.22)$; tumor subtype (HER2-amplified vs TN, $P=$ 0.17; HR-positive vs TN, $P=0.99$ ); or use of adjuvant chemotherapy $(P=0.45)$, adjuvant hormonal therapy $(P=0.40)$, or adjuvant radiation $(P=0.18)$. For NACT duration, the shortest duration ( $\leq 4$ months) had survival similar to those with more conventional lengths of treatment (1.0, 95\% CI $0.5-2.0$ versus $>4$ and $\leq 7$ patients, $P=0.89$ ), but patients with longer durations of NACT did worse ( $>7$ versus $>4$ and $\leq 7$, HR 1.9, 95\% CI 1.1-3.3, $P=0.018)$ (Additional file 1: Figure S1).

Boldface type represents those statistically significant values

\section{Discussion}

It is well established that pCR differs by therapeutic regimen and breast cancer subtype [3, 6, 14, 23], with patients who achieve a pCR demonstrating improved survival over those who do not. However, any influence of the NACT regimen employed on subsequent survival

Table 3 Hazard ratio of death, based on patient characteristics and treatments received

\begin{tabular}{|c|c|c|c|}
\hline Variable & Contrast & HR $(95 \% \mathrm{Cl})$ & $P$ value \\
\hline \multicolumn{4}{|c|}{ Multivariate landmark analysis $(n=642)$} \\
\hline \multirow[t]{3}{*}{ Regimen } & $A T+$ HER2 vs. A + T & $0.90(0.37-2.20)$ & 0.82 \\
\hline & $P C$ vs. $A+T$ & $1.28(0.55-2.98)$ & 0.56 \\
\hline & A no $T$ vs. $A+T$ & $1.25(0.47-3.35)$ & 0.66 \\
\hline \multirow[t]{2}{*}{ Age } & $21-40$ vs. $41-60$ & $2.00(1.05-3.82)$ & 0.036 \\
\hline & $>60$ vs. $41-60$ & $2.04(1.01-4.15)$ & 0.048 \\
\hline Postmenopausal & Yes vs. no & $0.82(0.43-1.59)$ & 0.57 \\
\hline \multirow[t]{4}{*}{ Stage } & $\| B$ vs. $\| A$ & $1.15(0.60-2.23)$ & 0.67 \\
\hline & $\| I A$ vs. $\| A$ & $0.73(0.24-2.15)$ & 0.56 \\
\hline & $\| \mathrm{II}$ vs. $\| \mathrm{A}$ & $2.06(0.87-4.88)$ & 0.099 \\
\hline & $\| I C$ vs. $\| A$ & $2.26(1.11-4.61)$ & 0.024 \\
\hline Grade & III vs. I/II & $0.67(0.35-1.28)$ & 0.22 \\
\hline \multirow[t]{2}{*}{ Subtype } & HER2-amplified vs. TN & $0.56(0.25-1.28)$ & 0.17 \\
\hline & HR vs. TN & $1.00(0.46-2.22)$ & 0.99 \\
\hline Adjuvant chemotherapy & Yes vs. no & $1.33(0.64-2.77)$ & 0.45 \\
\hline Adjuvant hormone therapy & Yes vs. no & $0.75(0.39-1.45)$ & 0.40 \\
\hline Adjuvant radiation treatment & Yes vs. no & $0.65(0.34-1.22)$ & 0.18 \\
\hline
\end{tabular}

Abbreviations: $A$ no $T$ Adriamycin-based therapy alone, $A+T$ Adriamycin plus taxane, $A T+H E R 2$ HER2-targeted therapy, $P C$ Provider choice, $H R$ Hormone receptor, TN Triple-negative breast cancer Italicized values are statistically significant 
in patients who have already achieved a pCR has not been explored previously. Data presented in the present study suggest that subsequent RFS among patients achieving $\mathrm{PCR}$ is not influenced by or associated with the regimen of NACT used to achieve that $\mathrm{pCR}$. The very high recurrence-free survival in this population supports the value of achieving a $\mathrm{pCR}$, but it also suggests that there may be limited value in continued systemic treatments in the postoperative setting for the $\mathrm{pCR}$ patient [15].

Because reaching a pCR, rather than the specific therapies used to achieve it, appears to be paramount, the early determination of treatment response will be an important research focus. Imaging alone [24-26] or targeted biopsy techniques $[27,28]$ may be effective tools in determining $\mathrm{pCR}$ after NACT. Imaging modalities that show promise in the assessment of chemotherapy response include ultrasound of both breast and axilla [24], dynamic contrast-enhanced magnetic resonance imaging (MRI) [29], MRI texture analysis [25], and trimodal imaging (ultrasound, mammography, and MRI) $[30,31]$. Shear-wave elastography of tumor stiffness before the start of chemotherapy has been associated with tumor response [26], and ${ }^{18} \mathrm{~F}$-fluorodeoxyglucose positron emission tomography/computed tomography (PET/ CT) has also been studied [32]. The latter work correlated metabolic response by PET/CT during NACT after as few as two cycles of chemotherapy to improved survival at 71-month follow-up. Such imaging may allow oncologists to determine who is responding to chemotherapy earlier and tailor treatment accordingly or even avoid surgery altogether [33, 34]. Such concepts are being explored through the NRG BR005 [31] trial and a National Institutes of Health Breast Cancer Steering Committee initiative [35].

The generalizability of a single-institution cohort to NACT patients treated with current regimens or outside the institution requires confirmation. First, these data lack molecular phenotypes. It is known that luminal A tumors are less sensitive to chemotherapy [1], but the relationship of residual tumor and survival holds true across constructed subtype [3], so the presented work should be negligibly disadvantaged by this. Second, although there was no statistical difference in RFS between NACT regimens, the wide CIs do not demonstrate true equivalence between regimens. This may reflect the relatively low event rate of recurrences after pCR ( 85 of 721 patients), similar to recent trial reports $[7,9,15]$, or it may be due to sample size. Third, it is difficult to interpret the effect of chemotherapy duration, owing to the retrospective nature of the analysis. NACT durations $>7$ months are likely due to NACT complications and unexpected delays of surgical treatment, but the shorterduration NACT patients present an interesting observation. Although it is promising that patients who achieved a pCR after only four cycles had survival similar to those receiving more conventional regimens, many patients at our institution are treated on study protocols or clinical trials, so this may be attributed to observational bias and, as such, should be interpreted cautiously. Regimen durations should be compared in future de-escalation trials. Last, these data were collected before taxotere/cyclophosphamide (TC) or paclitaxel+trastuzumab +/- pertuzumab $(\mathrm{TH}+/-\mathrm{P})$ were widely recognized as accepted neoadjuvant regimens, as indicated by the very few patients receiving these regimens, and highlighting the need now for further prospective trials to investigate the safe de-escalation of NACT.

Another consideration in this body of literature is that the relationship between NACT, pCR, and improved survival is complex. For example, the addition of taxanes led to increased pCR in NACT trials and also increased survival in adjuvant trials, suggesting an association between increased pCR and improved survival [36, 37]. However, the largest prospective cohort, NSABP B27, failed to show OS or DFS benefit despite a doubling of pCR. This influenced the pooled CTNeoBC analysis [1], which did not validate $\mathrm{pCR}$ as a surrogate endpoint for survival, suggesting that survival data after pCR should be interpreted with care.

Despite these limitations, this study does point to "atrisk" patient populations. Patients aged 21-40 years and stage IIIC patients had significantly increased risk of death on multivariate analysis. These higher-risk populations should be considered when making treatment decisions regarding additional adjuvant chemotherapy, and they merit future studies. Also, this study highlights that regardless of how you achieve pCR or RCB 0 (1), the outcome is the same. If a reliable imaging modality can be developed to predict treatment response after only a few cycles of chemotherapy, we may be able to administer taxane and HER2-targeted therapies only, and avoid adriamycin-based therapy, for example. Prospective trials minimizing neoadjuvant cytotoxic agents based on interim findings suggestive of $\mathrm{pCR}$ may be warranted.

\section{Conclusions}

These data suggest that postsurgical RFS among patients with pCR is not significantly influenced by the type of NACT prior to surgery. Because this work provides indirect data only, meta-analysis of randomized trial data should be explored to evaluate wider populations and larger sample sizes to minimize bias. However, the present study provides some reassurance for designing prospective trials aimed at personalization and de-escalation of neoadjuvant chemotherapy.

\section{Additional file}

Additional file 1: Figure S1. Five-year RFS by duration of neoadjuvant chemotherapy. (PPTX $72 \mathrm{~kb}$ ) 


\section{Funding}

This work was partially funded through the Nellie B. Connally Breast Center at the University of Texas MD Anderson Cancer Center.

\section{Availability of data and materials}

The datasets generated and/or analyzed during the present study are not publicly available, owing to patient identification, but they are available from the corresponding author on reasonable request.

\section{Authors' contributions}

AW has made substantial contributions to analysis and interpretation of data, has been involved in drafting the manuscript or revising it critically for important intellectual content, has given final approval of the version to be published, and has agreed to be accountable for all aspects of the work in ensuring that questions related to the accuracy or integrity of any part of the work are appropriately investigated and resolved. SIB has made substantial contributions to acquisition of data and analysis and interpretation of data, has been involved in revising the manuscript critically for important intellectual content, and has given final approval of the version to be published. $\mathrm{KH}$ has made substantial contributions to analysis and interpretation of data, has been involved in revising the manuscript critically for important intellectual content and has given final approval of the version to be published. AMT has been involved in drafting the manuscript and revising it critically for important intellectual content and has given final approval of the version to be published. NKI has made substantial contributions to conception and design, acquisition of data, and analysis and interpretation of data; has been involved in drafting the manuscript and revising it critically for important intellectual content; has given final approval of the version to be published; and has agreed to be accountable for all aspects of the work in ensuring that questions related to the accuracy or integrity of any part of the work are appropriately investigated and resolved. All authors read and approved the final manuscript.

\section{Ethics approval and consent to participate}

This study was approved by the University of Texas MD Anderson Cancer Center Institutional Review Board. The need for informed consent to participate was waived by the review board.

\section{Consent for publication}

Not applicable.

\section{Competing interests}

The authors declare that they have no competing interests.

\section{Publisher's Note}

Springer Nature remains neutral with regard to jurisdictional claims in published maps and institutional affiliations.

\section{Author details \\ 'Department of Surgical Oncology, Brigham and Women's Hospital, Dana Farber Cancer Institute, Boston, MA 02115, USA. ²Department of Breast Medical Oncology, University of Texas MD Anderson Cancer Center, 1155 Pressler Street CPB5.3540, Houston, TX 77030, USA. ${ }^{3}$ Department of Biostatistics, University of Texas MD Anderson Cancer Center, Houston, TX 77030, USA. ${ }^{4}$ Department of Breast Surgical Oncology, University of Texas MD Anderson Cancer Center, Houston, TX 77030, USA.}

Received: 3 October 2017 Accepted: 19 February 2018 Published online: 16 April 2018

\section{References}

1. Cortazar $P$, Zhang $L$, Untch $M$, et al. Pathological complete response and long-term clinical benefit in breast cancer: the CTNeoBC pooled analysis. Lancet. 2014;384:164-72

2. Symmans WF, Peintinger F, Hatzis $C$, et al. Measurement of residual breast cancer burden to predict survival after neoadjuvant chemotherapy. J Clin Oncol. 2007:25:4414-22.

3. Symmans WF, Wei C, Gould R, et al. Long-term prognostic risk after neoadjuvant chemotherapy associated with residual cancer burden and breast cancer subtype. J Clin Oncol. 2017;35:1049-60.

4. von Minckwitz G, Untch M, Blohmer JU, et al. Definition and impact of pathologic complete response on prognosis after neoadjuvant chemotherapy in various intrinsic breast cancer subtypes. J Clin Oncol. 2012; 30:1796-804.

5. Schneeweiss A, Chia S, Hickish T, et al. Pertuzumab plus trastuzumab in combination with standard neoadjuvant anthracycline-containing and anthracycline-free chemotherapy regimens in patients with HER2-positive early breast cancer: a randomized phase II cardiac safety study (TRYPHAENA). Ann Oncol. 2013;24:2278-84.

6. Schneeweiss A, Chia S, Hegg R, et al. Evaluating the predictive value of biomarkers for efficacy outcomes in response to pertuzumab- and trastuzumab-based therapy: an exploratory analysis of the TRYPHAENA study. Breast Cancer Res. 2014;16:R73.

7. Gianni L, Eiermann W, Semiglazov V, et al. Neoadjuvant chemotherapy with trastuzumab followed by adjuvant trastuzumab versus neoadjuvant chemotherapy alone, in patients with HER2-positive locally advanced breast cancer (the NOAH trial): a randomised controlled superiority trial with a parallel HER2-negative cohort. Lancet. 2010;375:377-84.

8. Baselga J, Bradbury I, Eidtmann $\mathrm{H}$, et al. Lapatinib with trastuzumab for HER2-positive early breast cancer (NeoALTTO): a randomised, open-label, multicentre, phase 3 trial. Lancet. 2012;379:633-40. A published erratum appears in Lancet. 2012;379:616

9. de Azambuja E, Holmes AP, Piccart-Gebhart M, et al. Lapatinib with trastuzumab for HER2-positive early breast cancer (NeoALTTO): survival outcomes of a randomised, open-label, multicentre, phase 3 trial and their association with pathological complete response. Lancet Oncol. 2014;15: 1137-46.

10. Wolmark N, Wang J, Mamounas E, et al. Preoperative chemotherapy in patients with operable breast cancer: nine-year results from National Surgical Adjuvant Breast and Bowel Project B-18. J Natl Cancer Inst Monogr. 2001;30:96-102.

11. Bear HD, Anderson S, Smith RE, et al. Sequential preoperative or postoperative docetaxel added to preoperative doxorubicin plus cyclophosphamide for operable breast cancer: National Surgical Adjuvant Breast and Bowel Project Protocol B-27. J Clin Oncol. 2006;24:2019-27.

12. Piccart-Gebhart MJ, Procter M, Leyland-Jones B, et al. Trastuzumab after adjuvant chemotherapy in HER2-positive breast cancer. N Engl J Med. 2005; 353:1659-72.

13. Romond EH, Perez EA, Bryant J, et al. Trastuzumab plus adjuvant chemotherapy for operable HER2-positive breast cancer. N Engl J Med. 2005:353:1673-84

14. Gianni L, Pienkowski T, Im YH, et al. Efficacy and safety of neoadjuvant pertuzumab and trastuzumab in women with locally advanced, inflammatory, or early HER2-positive breast cancer (NeoSphere): a randomised multicentre, open-label, phase 2 trial. Lancet Oncol. 2012:13:25-32.

15. Gianni L, Pienkowski T, Im YH, et al. 5-year analysis of neoadjuvant pertuzumab and trastuzumab in patients with locally advanced, inflammatory, or early-stage HER2-positive breast cancer (NeoSphere): a multicentre, open-label, phase 2 randomised trial. Lancet Oncol. 2016;17: 791-800.

16. Esserman $\amalg$, Berry DA, DeMichele $A$, et al. Pathologic complete response predicts recurrence-free survival more effectively by cancer subset: results from the I-SPY 1 TRIAL_CALGB 150007/150012, ACRIN 6657. J Clin Oncol. 2012:30:3242-9.

17. Bossuyt V, Provenzano E, Symmans WF, et al. Recommendations for standardized pathological characterization of residual disease for neoadjuvant clinical trials of breast cancer by the BIG-NABCG collaboration. Ann Oncol. 2015;26:1280-91.

18. U.S. Food and Drug Administration. Guidance for industry: pathological complete response in neoadjuvant treatment of high-risk early-stage breast cancer: use as an endpoint to support accelerated approval. Silver Spring, MD: U.S: Department of Health and Human Services, Food and Drug Administration, Center for Drug Evaluation and Research (CDER); 2014.

19. Prowell TM, Pazdur R. Pathological complete response and accelerated drug approval in early breast cancer. N Engl J Med. 2012;366:2438-41.

20. Hatzis C, Symmans WF, Zhang Y, et al. Relationship between complete pathologic response to neoadjuvant chemotherapy and survival in triplenegative breast cancer. Clin Cancer Res. 2016;22:26-33.

21. Turner EL, Dobson JE, Pocock SJ. Categorisation of continuous risk factors in epidemiological publications: a survey of current practice. Epidemiol Perspect Innov. 2010;7:9

22. Dafni U. Landmark analysis at the 25 -year landmark point. Circ Cardiovasc Qual Outcomes. 2011;4:363-71. 
23. Buzdar AU, Suman VJ, Meric-Bernstam F, et al. Fluorouracil, epirubicin, and cyclophosphamide (FEC-75) followed by paclitaxel plus trastuzumab versus paclitaxel plus trastuzumab followed by FEC-75 plus trastuzumab as neoadjuvant treatment for patients with HER2-positive breast cancer (Z1041): a randomised, controlled, phase 3 trial. Lancet Oncol. 2013;14:1317-25.

24. Candelaria RP, Bassett RL, Symmans WF, et al. Performance of mid-treatment breast ultrasound and axillary ultrasound in predicting response to neoadjuvant chemotherapy by breast cancer subtype. Oncologist. 2017;22:394-401.

25. Henderson S, Purdie C, Michie C, et al. Interim heterogeneity changes measured using entropy texture features on T2-weighted MRI at 3.0 T are associated with pathological response to neoadjuvant chemotherapy in primary breast cancer. Eur Radiol. 2017;27:4602-11.

26. Evans A, Rauchhaus $\mathrm{P}$, Whelehan $\mathrm{P}$, et al. Does shear wave ultrasound independently predict axillary lymph node metastasis in women with invasive breast cancer? Breast Cancer Res Treat. 2014:143:153-7.

27. Heil J, Kummel S, Schaefgen B, et al. Diagnosis of pathological complete response to neoadjuvant chemotherapy in breast cancer by minimal invasive biopsy techniques. Br J Cancer. 2015;113:1565-70.

28. Heil J, Schaefgen B, Sinn P, et al. Can a pathological complete response of breast cancer after neoadjuvant chemotherapy be diagnosed by minimal invasive biopsy? Eur J Cancer. 2016;69:142-50.

29. Li W, Arasu V, Newitt DC, et al. Effect of MR imaging contrast thresholds on prediction of neoadjuvant chemotherapy response in breast cancer subtypes: a subgroup analysis of the ACRIN 6657/I-SPY 1 TRIAL. Tomography. 2016;2. 378-87.

30. De Los Santos JF, Cantor A, Amos KD, et al. Magnetic resonance imaging as a predictor of pathologic response in patients treated with neoadjuvant systemic treatment for operable breast cancer: Translational Breast Cancer Research Consortium trial 017. Cancer. 2013;119:1776-83.

31. NRG Oncology. Assessing the Accuracy of Tumor Biopsies after Chemotherapy to Determine if Patients Can Avoid Breast Surgery. ClinicalTrials.gov, NCT03188393. https:/clinicaltrials.gov/ct2/show/NCT03188393. Accessed 3 Aug 2017.

32. Chen $\mathrm{S}$, Ibrahim NK, Yan Y, et al. Complete metabolic response on interim ${ }^{18}$ F-fluorodeoxyglucose positron emission tomography/computed tomography to predict long-term survival in patients with breast cancer undergoing neoadjuvant chemotherapy. Oncologist. 2017;22:526-34.

33. Schaefgen B, Heil J, Richter $\mathrm{H}$, et al. Detection and removal of ceramic clip markers from breast tissue by ultrasound-guided, vacuum-assisted minimally invasive biopsy in a turkey breast model. Ultrasound Med Biol. 2017;43:341-5.

34. van la Parra RF, Kuerer HM. Selective elimination of breast cancer surgery in exceptional responders: historical perspective and current trials. Breast Cancer Res. 2016;18:28.

35. Breast Cancer Steering Committee (BCSC), National Clinical Trials Network. 2015 Strategic priorities. https:/www.cancer.gov/about-nci/organization/ccct/ steering-committees/nctn/breast-cancer/bcsc-2015-strategic-priorities.pdf. Accessed 3 Aug 2017.

36. Green MC, Buzdar AU, Smith T, et al. Weekly paclitaxel improves pathologic complete remission in operable breast cancer when compared with paclitaxel once every 3 weeks. J Clin Oncol. 2005;23:5983-92.

37. Sparano JA, Wang M, Martino $S$, et al. Weekly paclitaxel in the adjuvant treatment of breast cancer. N Engl J Med. 2008;358:1663-71.

\section{Submit your next manuscript to BioMed Central and we will help you at every step:}

- We accept pre-submission inquiries

- Our selector tool helps you to find the most relevant journal

- We provide round the clock customer support

- Convenient online submission

- Thorough peer review

- Inclusion in PubMed and all major indexing services

- Maximum visibility for your research

Submit your manuscript at www.biomedcentral.com/submit
Biomed Central 